\title{
Dynamic model for hydro-turbine generator units based on a database method for guide bearings
}

\author{
Yong $\mathrm{Xu}^{\mathrm{a}, *}$, Zhaohui $\mathrm{Li}^{\mathrm{b}}$ and Xide Lai ${ }^{\mathrm{c}}$ \\ ${ }^{a}$ College of Water Resource and Hydropower, Sichuan University, Chengdu, Sichuan, China \\ ${ }^{\mathrm{b}}$ School of Hydropower and Information Engineering, Huazhong University of Science and Technology, Wuhan, \\ Hubei, China \\ ${ }^{\mathrm{c}}$ School of Energy and Environment, Xihua University, Chengdu, Sichuan, China
}

Received 9 March 2012

Revised 4 November 2012

Accepted 9 November 2012

\begin{abstract}
A suitable dynamic model of rotor system is of great significance not only for supplying knowledge of the fault mechanism, but also for assisting in machine health monitoring research. Many techniques have been developed for properly modeling the radial vibration of large hydro-turbine generator units. However, an applicable dynamic model has not yet been reported in literature due to the complexity of the boundary conditions and exciting forces. In this paper, a finite element (FE) rotor dynamic model of radial vibration taking account of operating conditions is proposed. A brief and practical database method is employed to model the guide bearing. Taking advantage of the method, rotating speed and bearing clearance can be considered in the model. A novel algorithm, which can take account of both transient and steady-state analysis, is proposed to solve the model. Dynamic response for rotor model of $125 \mathrm{MW}$ hydro-turbine generator units in Gezhouba Power Station is simulated. Field data from Optimal Maintenance Information System for Hydro power plants (HOMIS) are analyzed compared with the simulation. Results illustrate the application value of the model in providing knowledge of the fault mechanism and in failure diagnosis.
\end{abstract}

Keywords: Rotor dynamics, hydro-turbine generator units, radial vibration, modeling, simulation

\section{Introduction}

Large hydro-turbine generator units play important roles in power network, special attentions need to be paid to ensure their stability and safety in the process of operation. Vibration of shaft system is one of the most important factors affecting the stability and safety. Shaft system of a unit might be submitted to axial, torsional and radial vibration, among which radial vibration attracts the most attention [1]. An applicable simulation model of radial vibration is influential for the following aspects: the comprehension of mechanism to the oscillation phenomenon, the failure diagnosis and condition-based maintenance of units. The shaft system of a unit can be modeled by a finite element (FE) method or a transfer matrix method. As it is known that a FE method would bring more accurate results than a transfer matrix method, the FE method is adopted to model the shaft system. However, one practical

\footnotetext{
*Corresponding author: Yong Xu, College of Water Resource and Hydropower, Sichuan University, Chengdu 610065, Sichuan, China. E-mail: hustxyong@gmail.com.
} 


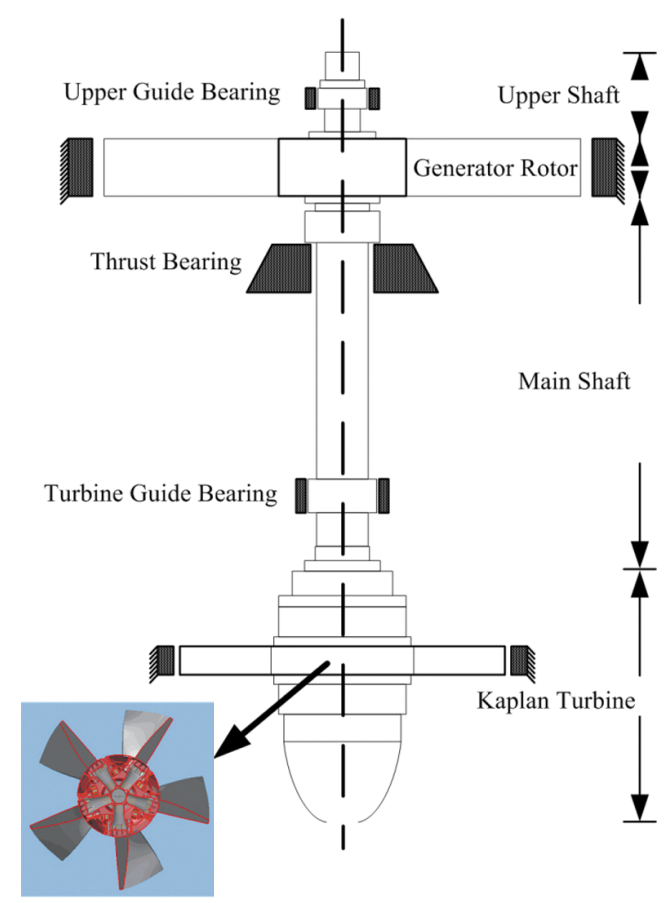

Fig. 1. Sketch of shaft system for 125 MW hydro-units in Gezhouba Hydropower Plant.

problem is that almost all the boundary conditions and exciting forces are field associated. Numerical methods such as finite element method (FEM) are useful to obtain a numerical solution for the field associated problems, but the time for the calculation is so long that a FE rotordynamic model with such boundary model is too time-consuming for practical application [2]. Therefore, proper simplification for the analysis of boundary conditions is urgently needed for an applicable simulation model.

As one of the most important boundary conditions, the force produced by guide bearing is of prime importance to calculate the dynamic response. A large number of papers have been published concerned with this topic [3]. However, since there is no stationary position in a vertical machine, the linear dynamic coefficients of guide bearings cannot be used [4]. Methods coped with this problem have been presented in previous literature. The methods mentioned in previous literature may be classified into the following two categories:

a) Making some assumptions such as misalignment and preload, then the linear dynamic coefficients could be used [5].

b) Iteratively solve the Reynolds equation with numerical method in a step by step way until the solution is accurate enough $[6,7]$.

Method a) is suitable for a certain condition like natural frequency analysis or dynamic response of steady-state analysis, but it might be oversimplified for the dynamic response of transient analysis. Method b) is suitable for the analysis of system because of its high accuracy. However, it is so time-consuming that the extensively use of this method is not advised. Either oversimplified or time-consuming method to analyze the guide bearing is not suitable for a simulation model. A brief and practical nonlinear guide bearing model may be prominent for the simulation of shaft system for large hydro-unit.

\section{Modeling a large hydro-unit}

A Kaplan type of large hydro-unit with vertical arrangement in Gezhouba, Fig. 1, is modeled. It has a nominal power of $125 \mathrm{MW}$ at a rated rotational speed of $62.5 \mathrm{rpm}$ and a runaway speed of $140 \mathrm{rpm}$. The main shaft is hollow with inner diameter of $1290 \mathrm{~mm}$ and outer diameter of $1620 \mathrm{~mm}$. The diameters of generator rotor and water turbine 


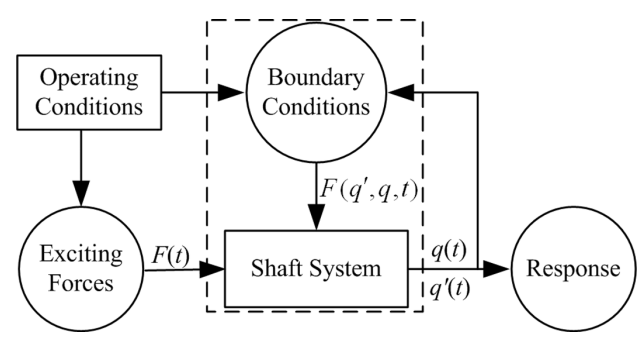

Fig. 2. Logic structure of radial vibration simulation model.

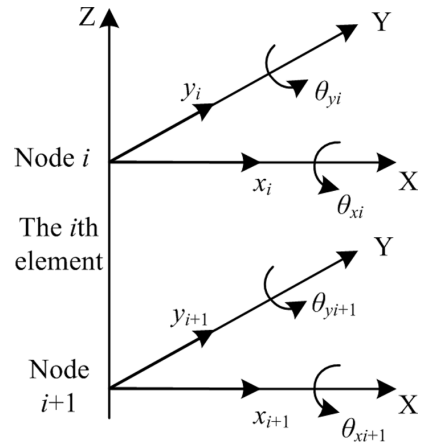

Fig. 3. Coordinates for the $i$ th element.

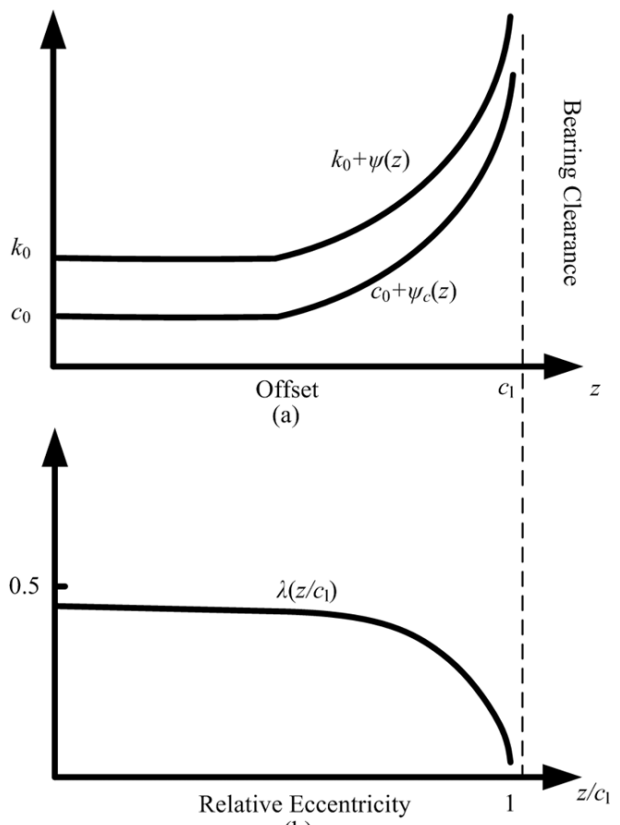

(b)

Fig. 4. Schematic diagram of (a) $k_{0}+\Psi(z), c_{0}+\Psi_{c}(z)$ vs. $z$ and (b) $\lambda\left(z / c_{1}\right)$ vs. $z / c_{1}$.

are $14.96 \mathrm{~m}$ and $10.2 \mathrm{~m}$ respectively. The thrust bearing is arranged below the rotor with 18 pads, which could be modeled as an equivalent coefficient of torsional rigidity in radial vibration model when the elastic oil tanks work normally [8]. Two tilting pad guide bearings are fixed along the shaft above the rotor and the runner respectively, the distance of them is $12.9 \mathrm{~m}$.

\subsection{Logic structure of the model}

The simulation model consists of shaft system, boundary conditions, exciting forces and operating conditions. Shaft system, including upper shaft, generator rotor, main shaft and water turbine, is the rotational part of the hydro-unit and the most important component of the model. Boundary conditions, including guide bearings, thrust bearing etc., are related to the relative displacements and velocities of the journal. That is, feedback exists between boundary conditions and their responses. The boundary conditions might be considered as part of the shaft system. Exciting forces due to malfunction or originated from specific operating conditions are other key factors that affect the dynamic response. Operating conditions such as rotational speed, load and excitation current, have great effects on boundary conditions and exciting forces as well. As is known that, bearing force and unbalanced force are related to the rotational speed, hydraulic force is related to the load while unbalanced magnetic pull is related to the field current. To build an applicable simulation model, all the factors mentioned above should be concerned in the simulation model. The logic structure of the model is shown in Fig. 2.

\subsection{Equations of motion}

To obtain a satisfactory model of radial vibration of shaft system for the hydro-unit, finite element method is employed. The shaft system is divided into several elements, and each element equation is formed separately. Global equation of motion is then obtained by superposition of these equations.

The shaft is modeled as rotational Timoshenko beam elements [9]. Compared with Bernoulli beam element, gyroscopic effects and shear deformations are considered in Timoshenko beam element. Shear coefficient for rotational Timoshenko beam given by Hutchinson [10] is adopted here. There are two nodes in one element, and each node 
has four degrees of freedom of motion, two displacement degrees of freedom (dofs), $x, y$, and two distortion angle ones, $\theta_{x}, \theta_{y}$. The element equation of rotational Timoshenko beam takes the following form

$$
M_{e} \ddot{q}_{e}(t)+G_{e} \dot{q}_{e}(t)+K_{e} q_{e}(t)=F_{e}(t)
$$

where the subscript $e$ denotes the element,

$M_{e}$ is element mass matrix,

$G_{e}$ is element gyroscopic matrix,

$K_{e}$ is element stiffness matrix,

$q_{e}$ is an arrangement of eight generalized dofs of two nodes in an element. It can be expressed as $\left[x_{i}, y_{i}, \theta_{x i}, \theta_{y i}\right.$, $\left.x_{i+1}, y_{i+1}, \theta_{x i+1}, \theta_{y i+1}\right]^{T}$ for the $i$ th element (shown in Fig. 3).

$F_{e}$ are exciting forces assumed only act on nodes.

Generator rotor is modeled as a rigid disc element where the stiffness is neglected. The element equation of rigid disc element can be simplified as following:

$$
M_{d} \ddot{q}_{d}(t)+G_{d} \dot{q}_{d}(t)=F_{d}(t)
$$

where the subscript $d$ denotes the disc,

$M_{d}$ is a symmetric matrix that contains the inertia $m$ and the transverse mass moment of inertia $J_{d}$ of the disc,

$G_{d}$ is a skew-symmetric matrix contains the polar mass moment of inertia $J_{p}$ of the disc,

$q_{d}$ is an arrangement of four generalized dofs of the node in a rigid disc element,

$F_{d}$ are exciting forces assumed only act on the node of disc.

Water turbine is modeled as rotational Timoshenko beam elements with different outer diameters, where runner blades and added mass due to water flow are modeled as point mass acting on the corresponding node. The dynamic added mass within the turbine is set to be $20 \%$ to $40 \%$ mass of water in the runner chamber according to reference [11]. It is known that equivalent mass due to water flow will increase the damping of the system, and we can easily consider the influence of this damping by adding the equivalent damping coefficients to the system damping matrix. However, as we are unable to determine the size of this equivalent damping coefficients, the present model only considers the influence of the guide bearing damping coefficients.

Dynamic characteristics of boundary conditions are always nonlinear and depend on displacement, velocity, rotational speed etc.. It is not easy to figure out a simple expression to describe the dynamic characteristics. Hence, numerical methods are often used to solve the Reynolds equation and obtain the dynamic characteristics. This is such a time-consuming way in transient analysis of system that simplification is urgently needed. Detailed discussion of the simplification will be presented in section three of the paper. Under some specific conditions, linearization could be used to simplify the analysis, and then the boundary conditions could be modeled as damping spring.

$$
F_{b}\left(\dot{q}_{b}, q_{b}, t\right)=C_{b} \dot{q}_{b}(t)+K_{b} q_{b}(t)
$$

where the subscript $b$ denotes the boundary conditions,

$C_{b}$ is the equivalent damping matrix,

$K_{b}$ is the equivalent stiffness matrix,.

$q_{b}$ is an arrangement of four generalized dofs at the boundary node,

$F_{b}$ are the forces acting on nodes due to the dynamic characteristics of boundary conditions.

Another classical form of the expression is as following:

$$
\left[\begin{array}{l}
F_{x} \\
F_{y}
\end{array}\right]=\left[\begin{array}{ll}
c_{x x} & c_{x y} \\
c_{y x} & c_{y y}
\end{array}\right]\left[\begin{array}{l}
\dot{x} \\
\dot{y}
\end{array}\right]+\left[\begin{array}{ll}
k_{x x} & k_{x y} \\
k_{y x} & k_{y y}
\end{array}\right]\left[\begin{array}{l}
x \\
y
\end{array}\right]
$$

where $x, y$ are the two displacement dofs and $c_{x x}, c_{x y}, c_{y x}, c_{y y}, k_{x x}, k_{x y}, k_{y x}, k_{y y}$ are the eight bearing coefficients, which are very useful to the analysis of rotor system.

Exciting forces could be from mechanical, hydraulic and electromagnetic origin. Unbalanced magnetic pull (UMP) is an electromagnetic originated force due to the non-uniform air-gap flux density around the rotor circumference. An analytical method has been proposed to calculate the unbalanced magnetic pull, which is taking 
account of field current and saturation effects [12]. Taking advantage of the method, effect of field current on vibration could be investigated. Compared with the traditional method which treat the UMP as a negative magnetic stiffness, this analytical method may contribute to more accurate results of UMP, and more factors on UMP could be concerned. Hydraulic force could be classified into three types: constant force, periodic force and stochastic force. Only the periodic force is considered in this study, and the effect of load on hydraulic force has not been included yet. The hydraulic force would be explored much further in future research. Eccentric mass of generator rotor may give rise to an unbalanced force of mechanical origin. It has the following form:

$$
F(t)=m r \Omega^{2} e^{j \Omega t+\varphi}
$$

where $m$ is mass of the rotor, $r$ is the offset of centroid, $\Omega$ is the rotational speed and $\varphi$ is the initial phase of the offset. It can be seen in Eq. (4) that the unbalanced force is related to the rotational speed $\Omega$.

Superposing from Eq. (1) to Eq. (4) by nodes, the global equation of motion is constructed as follow:

$$
M \ddot{q}(t)+C \dot{q}(t)+K q(t)=F(t)+F(\dot{q}, q, t)
$$

where

$M$ is the mass matrix of system,

$C$ is the gyroscopic matrix added with damping matrix of system

$K$ is the stiffness matrix of system,

$q$ is an arrangement of all generalized dofs of the nodes.

$F(t)$ are the time dependent forces like unbalanced forces etc.,

$F(\dot{q}, q, t)$ are the forces depending on the displacements and velocities like bearing forces and seal forces etc..

It is easy to find in Eq. (5) that the equation of motion of the system is a linear system with local nonlinearity in several nodes.

\section{Analyzing the guide bearing}

Dynamics of the guide bearings have great effects on the dynamic response of the system. Neither oversimplified nor time-consuming method to analyze the guide bearing is suitable for a simulation model. In this section, a brief and practical method to analyze the guide bearing is introduced. Taking advantage of the method, an applicable simulation model could be obtained, and the operating conditions could be considered in the model.

\subsection{Bently/Muszynska model}

Based on numerous observations from field test, a simple model for fluid related problems was proposed by Muszynska and Bently at BRDRC [13], which is also suitable for fluid lubricated bearings. Oil surrounding the rotor was treated as a mass of vibration in the model, showing equivalent mass, stiffness and damping. Nevertheless, the mass of vibration was rotating with the shaft system at a rotational speed of $\lambda \Omega$. The nonlinear model of bearing has the following form:

$$
\left[\begin{array}{c}
F_{x} \\
F_{y}
\end{array}\right]=\left[\begin{array}{cc}
k_{0}+\psi(z) & \lambda(z) \Omega\left(c_{0}+\psi_{c}(z)\right) \\
-\lambda(z) \Omega\left(c_{0}+\psi_{c}(z)\right) & k_{0}+\psi(z)
\end{array}\right]\left[\begin{array}{l}
x \\
y
\end{array}\right]+\left[\begin{array}{cc}
c_{0}+\psi_{c}(z) & \\
& c_{0}+\psi_{c}(z)
\end{array}\right]\left[\begin{array}{l}
\dot{x} \\
\dot{y}
\end{array}\right]
$$

where $k_{0}$ is the equivalent stiffness and $c_{0}$ is the equivalent damping when offset $z$ equals zero. $\lambda$ is the fluid circumferential average velocity ratio introduced by Muszynska, which is decreased with the offset $z$. Functions of $k_{0}+\Psi(z), c_{0}+\Psi_{c}(z)$ and $\lambda(z)$ (shown in Fig. 4) demonstrated the relationship between equivalent stiffness, equivalent damping, and fluid circumferential average velocity ratio with offset $z$ respectively. Substituting Eq. (6) with $\lambda=\lambda(z), k=k_{0}+\Psi(z)$ and $c=c_{0}+\Psi_{c}(z)$, following form is obtained:

$$
\left[\begin{array}{c}
F_{x} \\
F_{y}
\end{array}\right]=\left[\begin{array}{cc}
k & \lambda \Omega c \\
-\lambda \Omega c & k
\end{array}\right]\left[\begin{array}{l}
x \\
y
\end{array}\right]+\left[\begin{array}{c}
c \\
c
\end{array}\right]\left[\begin{array}{l}
\dot{x} \\
\dot{y}
\end{array}\right]
$$

Comparing Eq. ( $\left.3^{\prime}\right)$ with Eq. (7), it may be not hard to see that Eq. (7) is a special case of Eq. ( $\left.3^{\prime}\right)$. It is shown in Fig. 4 that the dynamic characteristics are almost constant when offset is small, and as offset becomes larger, nonlinearity appears. 
Table 1

Influence of $\varepsilon$ on the simulation results (UGB). $N I$ is the iteration number and $k_{e}$ is the equivalent stiffness

\begin{tabular}{lllllllll}
\hline$N / \mathrm{rpm}$ & \multicolumn{7}{l}{62.5 (rated speed) } & \multicolumn{7}{l}{140 (runaway speed) } \\
\hline$\varepsilon / \mu \mathrm{m}$ & 1 & 3 & 5 & 10 & 3 & 5 & 10 & 20 \\
$N I$ & 292 & 56 & 40 & 1 & 1754 & 1073 & 790 & 345 \\
$k_{e} / \times 10^{9}$ & 1.11 & 1.11 & 1.11 & 1.11 & $1.24-1.37$ & $1.21-1.45$ & $1.13-1.57$ & $1.11-1.91$ \\
\hline
\end{tabular}

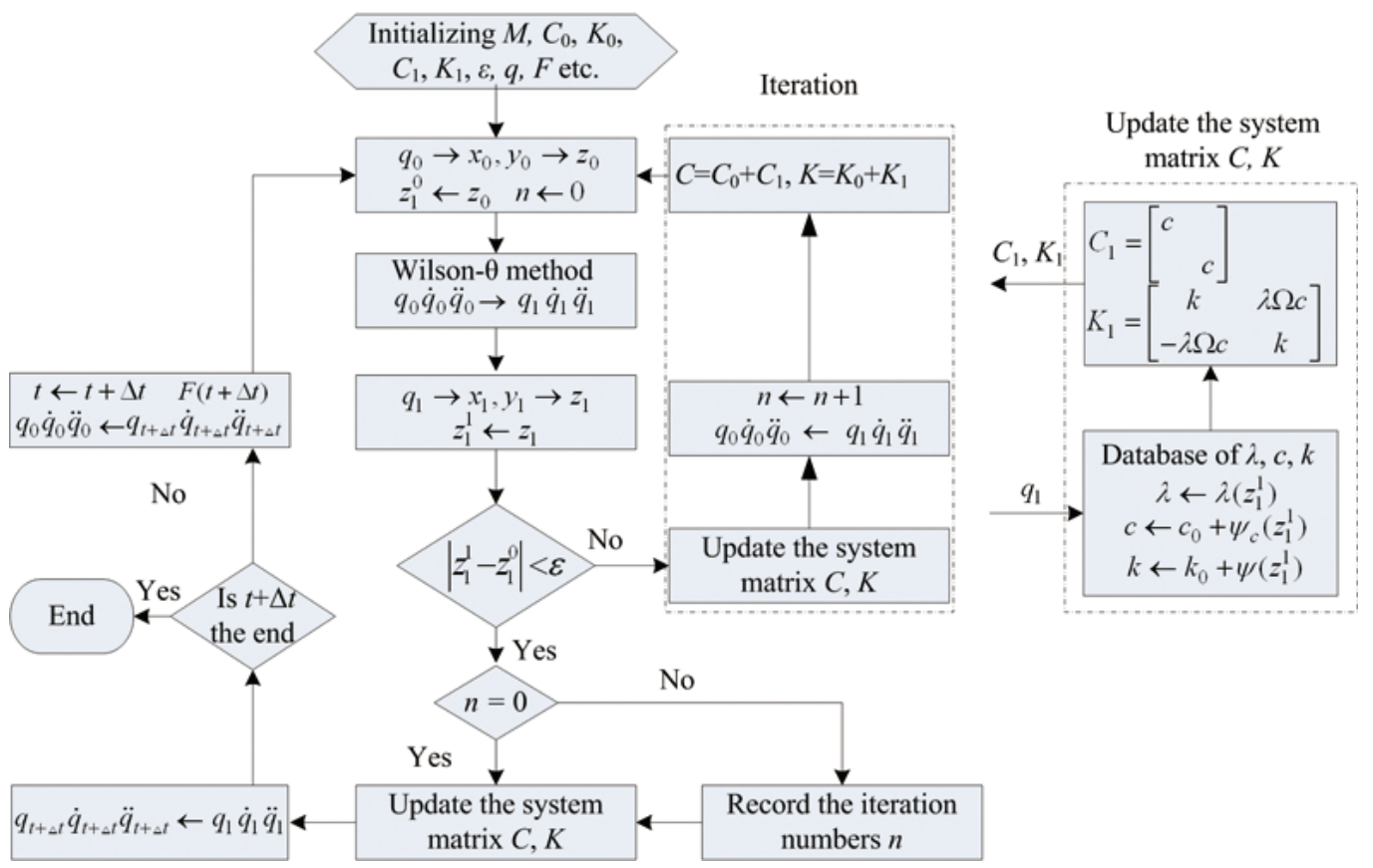

Fig. 5. Flow chart of the algorithm. The left arrows $\leftarrow$ in the box denotes assigning a value to the left and the right arrows $\rightarrow$ denotes calculating from the left. $x, y$ are the two displacement dofs in $q$ and $z=\sqrt{x^{2}+y^{2}}$.

\subsection{Database method}

As it is not easy to obtain the functional form of $\Psi(z), \Psi_{c}(z)$ and $\lambda(z)$, a database of $\lambda(z), c(z)$ and $k(z)$ with $z$ could be built up instead. Relationship between bearing coefficients $c_{x x}, c_{x y}, c_{y x}, c_{y y}, k_{x x}, k_{x y}, k_{y x}, k_{y y}$ and coefficients $\lambda, k, c$ in $\mathrm{B} / \mathrm{M}$ model has the following form:

$$
\lambda=\frac{k_{x y}-k_{y x}}{\Omega\left(c_{x x}+c_{y y}\right)}, \quad c=\frac{c_{x x}+c_{y y}}{2}, \quad k=\frac{k_{x x}+k_{y y}}{2}+\frac{k_{x y}-k_{y x}}{4} \frac{c_{x y}-c_{y x}}{c_{x x}+c_{y y}}
$$

Taking the advantages of the relationship, fluid bearing data from handbooks, field test or numerical methods [14] could be used with Eq. (8) to build up the database. Interpolation is used to enlarge the database where to no reference data could be referred.

Once the database of the coefficients for B/M model is obtained, database method could be used to add the B/M model in the simulation model of radial vibration. Assuming that the shaft system is concentric with stator in the initial state, offset $z$ could be calculated by the response displacement $x, y$ and coefficients for B/M model could be obtained, and then the guide bearing force could be updated by Eq. (7).

\section{An algorithm solving the model}

To solve the equations of motion of the system with local nonlinearity, an algorithm combines Wilson- $\theta$ method with iteration is proposed, which takes full advantages of the database method. $\varepsilon$ is a given parameter determining 


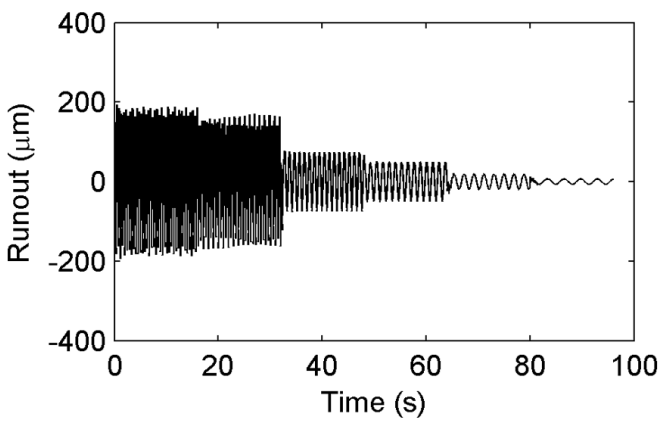

(a)

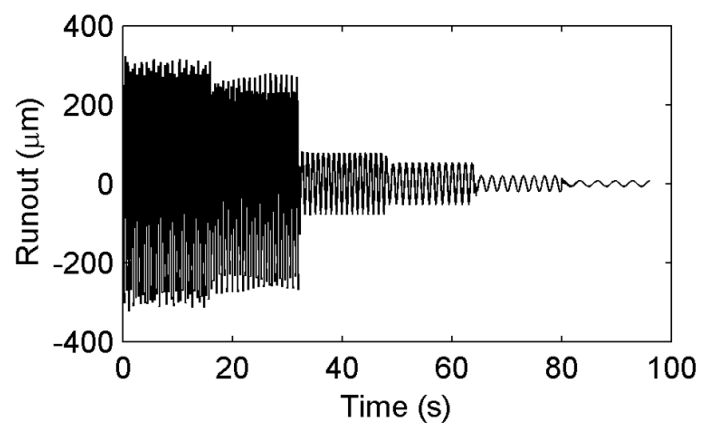

(b)

Fig. 6. Response of the simulation at UGB direction X, (a) Our model and (b) A constant coefficients model.

whether to enable the iteration or not. If the variation of shaft runout, which could be extracted from dynamic response of the system, is greater than $\varepsilon$, the system is considered to be in a transient process and iteration is enabled to ensure the accuracy of calculation. Otherwise, the system is considered to be in a steady-state process and iteration is disabled. A detailed flow chart of the algorithm is shown in Fig. 5. It is almost the same efficiency of the algorithm as one solving a linear model while steady-state process is calculated, and in a transient process, good accuracy could be obtained.

As is shown in Table 1, smaller $\varepsilon$ tends to increase the iteration number, NI, accuracy and calculation time, while an oversized $\varepsilon$ may lead to errors at transient process. Proper $\varepsilon$ could enable iteration to ensure accuracy at transient process and disable iterations to ensure the calculation speed in the steady-state process. It can be concluded from Table 1 that dynamic bearing coefficients that are suitable for a certain operating condition (like rated speed) would not be suitable any more for another operating condition (like runaway speed). Hence, using a linear model with constant dynamic coefficients to simulate the dynamic response of hydro-units may lead to errors.

\section{Simulation results}

Dynamic response at nodes of upper guide bearing (UGB) and turbine guide bearing (TGB) are extracted for analysis, where the vibration sensors are mounted. This would be useful for the comparison of simulation results and the field data. The field data analyzed here are obtained from Optimal Maintenance Information System for Hydro power plants (HOMIS) [15].

\subsection{Simulation of rotating speed test}

Generally, rotating speed test is used to decide whether unbalance malfunction exists. Simulation of rotating speed test for a $125 \mathrm{MW}$ Kaplan hydro unit is presented, where the unbalanced forces, originated from the unbalance malfunction, are exerted at the node of the rotor. The unit is set to operate at the rotational speed of $140 \mathrm{rpm}, 2 \times n_{r}$, $1 \times n_{r}, 0.8 \times n_{r}, 0.5 \times n_{r}$ and $0.3 \times n_{r}$ in turn, where $n_{r}$ is the rated speed $(62.5 \mathrm{rpm})$ and $140 \mathrm{rpm}$ is the runaway speed which could not be allowed to operate in actual units. Each speed is operated for $16 \mathrm{~s}$. Mass of the rotor, $m$, is $630000 \mathrm{~kg}$ and the offset of centroid $r$ is assumed to be $5 \mathrm{~mm}$. $\varepsilon$ is chosen to be $5 \mu \mathrm{m}$. The response of calculating results is shown in Fig. 6(a). It is found that the variation of magnitude of response is in accordance with the variation of speed. As a comparison, response of a constant coefficients model is presented in Fig. 6(b). The comparison highlights the nonlinear effect of our model. Figure 7 shows the time history and their spectra of the actual readings when the unit is under rotating speed test. Due to the limitation of field test, only speed of $1 \times n_{r}$ and $0.8 \times n_{r}$ are available at the field data. Taking the advantages of the model, results that cannot be observed at an actual hydro-unit could be obtained. When the field data are analyzed compared with the simulation results, more operating characteristics of the unit could be obtained.

To validate the effectiveness of the algorithm, we record the iteration number and equivalent stiffness at each step size of calculating time. Figure 8 shows the variation of iteration number and equivalent stiffness in the rotating speed 


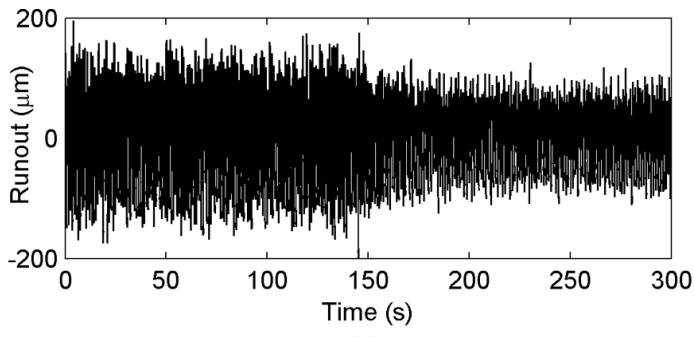

(a)

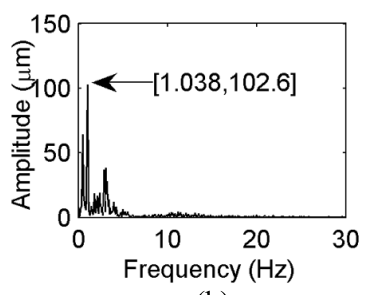

(b)

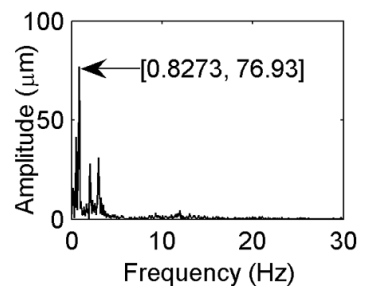

(c)

Fig. 7. Field data under rotating speed test at UGB direction $\mathrm{X}$, (a) Time history; (b) Spectra of the first half part (1 $\left.\times \mathrm{n}_{r}\right)$; (c) Spectra of the second half part $\left(0.8 \times \mathrm{n}_{r}\right)$.

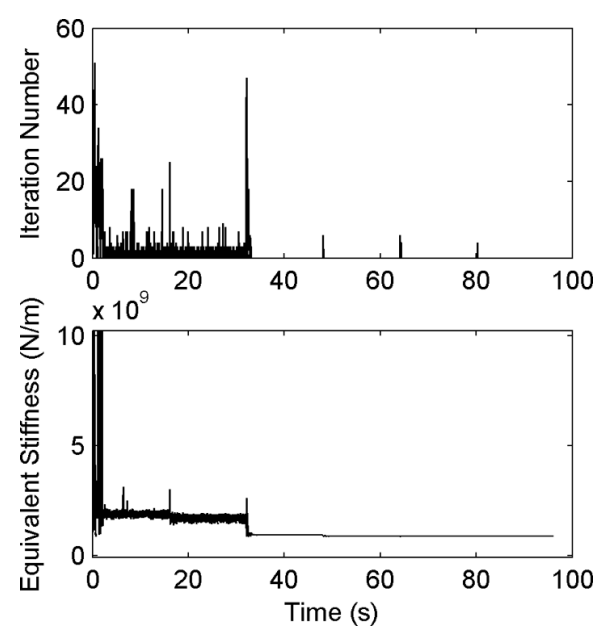

Fig. 8. The variation of iteration number and equivalent stiffness in the simulation of rotating speed test.

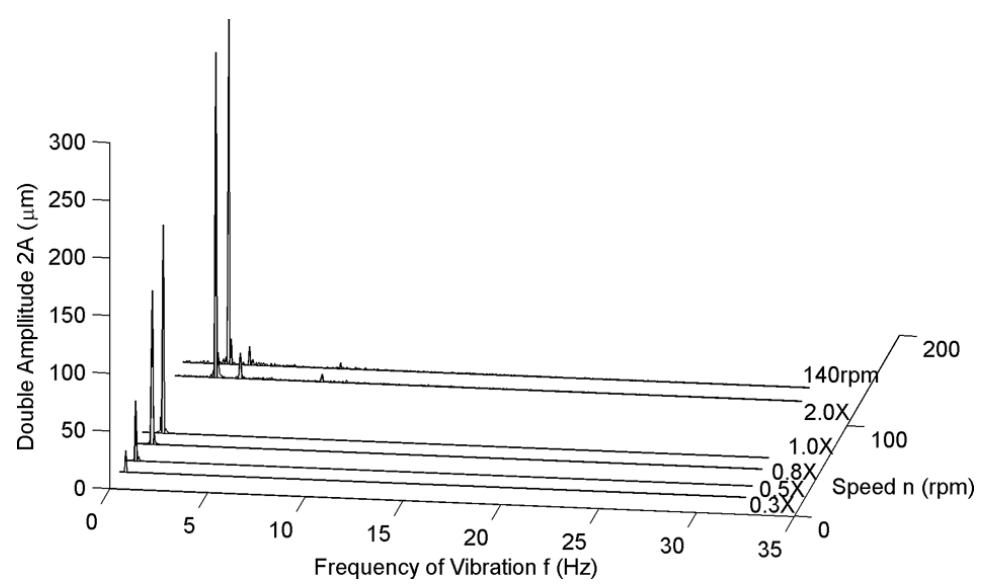

Fig. 9. Waterfall plot for double amplitude spectrum at UGB direction X.

test simulating. It could be seen that iterations only take place when the unit is operated at over speed condition or when the speed is changed, and equivalent stiffness coefficients vary with the variation of speed timely. This indicates that the algorithm is effective. Figure 9 shows a waterfall plot about rotational speed, frequency of vibration and double amplitude of vibration. The trends of the basic frequencies are clearly described.

\subsection{Effect of bearing clearance on vibration}

As is shown in Fig. 7, the peak-to-peak value of actual unit is a bit larger than that of the specified mounting clearance of the upper guide bearing $(320 \mu \mathrm{m})$. Seen from the long term field data at HOMIS, this situation had been lasted for several months. It could be concluded from operating experience that the mounting clearance had already been enlarged [16]. Figure 10 shows a comparison of dynamic response for specified bearing mounting clearance and enlarged bearing mounting clearance. In Fig. 10, the vibration amplitude is shown as a function of rotational speed, and the markers show actual readings from the field test. For purposes of comparison, field data are selected when (a) the unit is undergoing an unbalance malfunction (shown in Fig. 8) and (b) after an overhaul.

It can be seen from Fig. 10 that dynamic response for specified mounting clearance is smaller than that for enlarged mounting clearance. The reason is that the increased mounting clearance leads to a decrease in dynamic bearing coefficients, and the dynamic response is therefore enlarged. Good agreement is obtained from the simulation results and the actual readings. 
Table 2

Values of damping for bearings in the simulation $\left(\mathrm{Nm}^{-1} \mathrm{~s}\right)$

\begin{tabular}{llccccc}
\hline & z/c 1 & 0.1 & 0.3 & 0.5 & 0.7 & 0.9 \\
\hline \multirow{2}{*}{ UGB } & Properly damped & $1.95 \mathrm{e} 07$ & $7.79 \mathrm{e} 07$ & $3.12 \mathrm{e} 08$ & $2.10 \mathrm{e} 09$ & $7.79 \mathrm{e} 10$ \\
& Under damped & $4.87 \mathrm{e} 05$ & $1.95 \mathrm{e} 06$ & $7.79 \mathrm{e} 06$ & $5.26 \mathrm{e} 07$ & $1.95 \mathrm{e} 09$ \\
\multirow{2}{*}{ TGB } & Properly damped & $4.19 \mathrm{e} 07$ & $1.68 \mathrm{e} 08$ & $6.71 \mathrm{e} 08$ & $4.53 \mathrm{e} 09$ & $1.68 \mathrm{e} 11$ \\
& Under damped & $1.05 \mathrm{e} 06$ & $4.19 \mathrm{e} 06$ & $1.68 \mathrm{e} 07$ & $1.13 \mathrm{e} 08$ & $4.19 \mathrm{e} 09$ \\
\hline
\end{tabular}

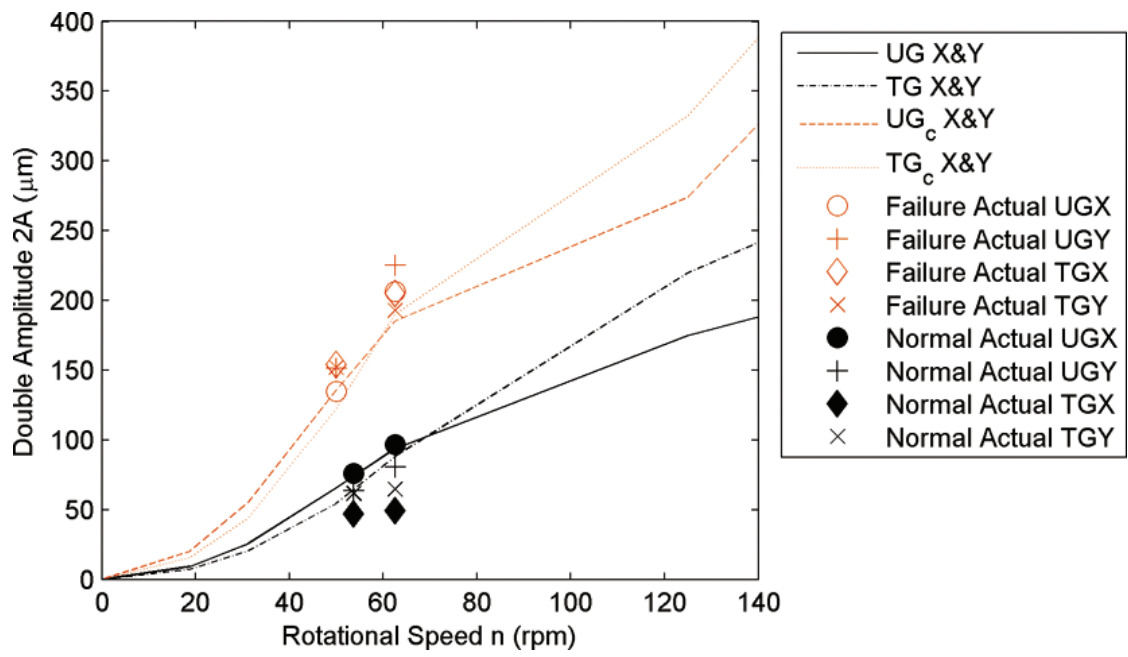

Fig. 10. Comparison of simulation results for specified bearing mounting clearance (UG and TG) and enlarged bearing mounting clearance (UG and TG enlarged). Markers show the actual readings from field test.

\subsection{Effect of bearing damping on vibration}

Figure 11 shows the comparison of amplitude spectrum for UGB at direction X. As is shown in Fig. 11(a), the unit had been suffering from a secondary frequency between second harmonic generation (SHG) and third harmonic generation (THG) in amplitude spectrum for some time. Simulations were constructed in order to interpret the reason. When the guide bearings were underdamped, the same phenomenon was observed from simulation results in Fig. 11(b), and when the guide bearings were damped properly, the secondary frequency disappeared in Fig. 11(c). Table 2 shows the values of damping for bearings in the simulation. To obtain a better comparison, damping values for under damped bearings are assumed one fortieth of that for properly damped bearings.

The first critical speed of the rotor system is calculated to be $179.4934 \mathrm{rpm}$ (critical frequency is $2.9916 \mathrm{~Hz}$ ) by using the bearing coefficients of simulation at the moment, while the secondary frequency in the amplitude spectrum of direction X at UGB is $2.988 \mathrm{~Hz}$, which is very close to the first critical frequency. This is an implication for the unit that the secondary frequency in amplitude spectrum is the first critical frequency, and the appearance of first critical frequency $2.954 \mathrm{~Hz}$ may be attributed to the underdamped guide bearings.

The first critical speed of a hydro-unit is designed to be at least $20 \%$ larger than the runaway speed (140 rpm), thus the first critical speed of the unit is designed to be larger than $168 \mathrm{rpm}$. The value of $177.24 \mathrm{rpm}$ satisfies the design requirement. It may verify the implication from another point of view.

\section{Discussion}

The greatest advantage of the model presented in this paper is that operating conditions rotating speed could be considered and the dynamic responds can be calculated in an acceptable computing time. This is due to the brief 


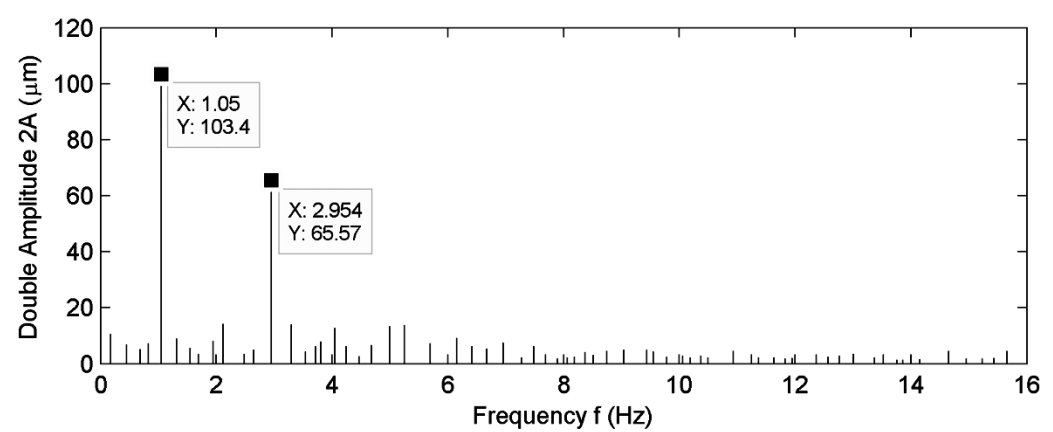

(a)

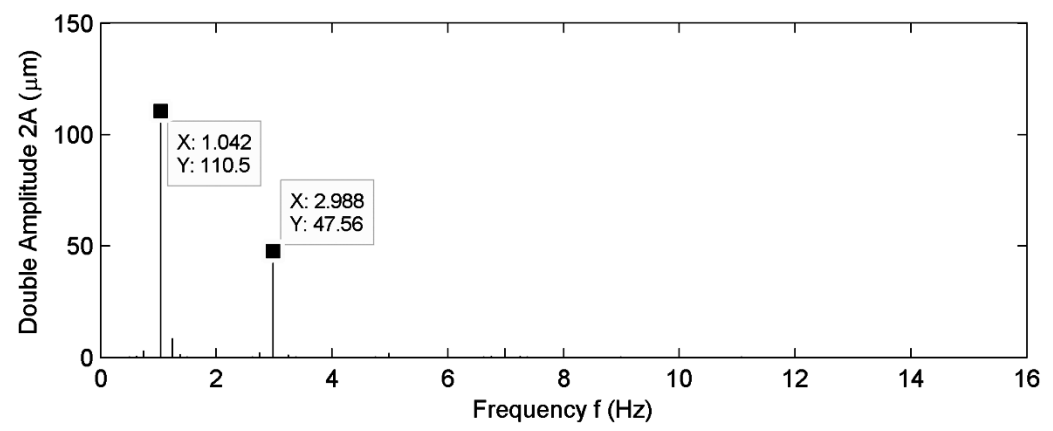

(b)

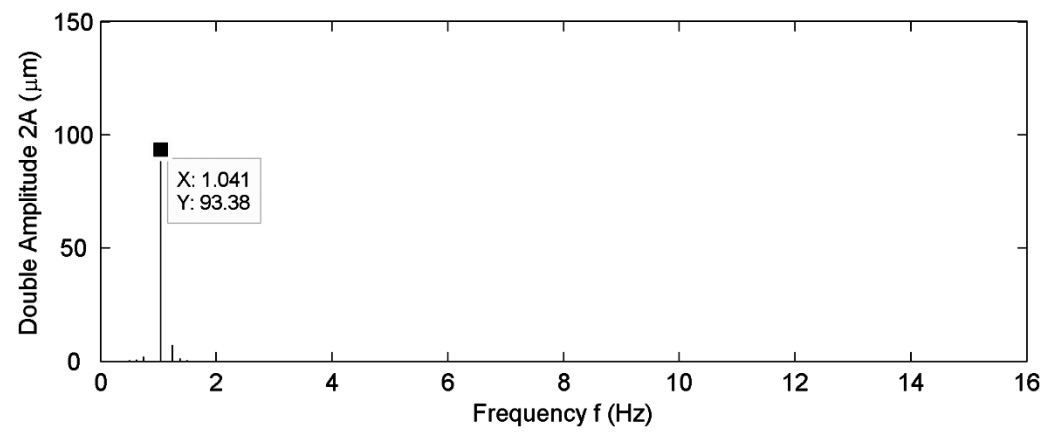

(c)

Fig. 11. Comparison of amplitude spectrum for UGB at direction X. (a) Field data; (b) Simulation result of underdamped bearings; (c) Simulation result of damped bearings.

and practical database method modeling the guide bearing force. The drawback of the method is the decrease of accuracy. This might be compensated by a good database of the method.

Other operating conditions like field current and load should also be considered properly in the model. An analytical method has been proposed by the authors to calculate the unbalanced magnetic pull taking account of field current and saturation effects. Hydraulic force and the effect of load on it have not been discussed in this study, which will be discussed in a future work. Although not all the factors are considered, the current simulation model still shows its application value. If all the factors are considered properly, a more applicable simulation model could be obtained.

\section{Conclusions}

A simulation model of radial vibration for large hydro-unit has been described. FEM was employed to model the shaft system and a brief and practical database method was proposed to model the guide bearing force. Taking 
advantage of the simplified boundary model, operating conditions, important factors affecting the dynamic response, had been considered in the model. An algorithm taking account of both transient and steady-state analysis was proposed to solve the model.

Rotating speed test for a 125 MW Kaplan hydro unit was simulated. Compared with the simulation results, the field data under rotating speed test from HOMIS were analyzed. From the comparison, it could be concluded that the mounting clearance of upper guide bearing had already been enlarged when the field data were recorded; and the appearance of secondary frequency between SHG and THG in amplitude spectrum of field data may be attributed to the under-damped guide bearings.

\section{Acknowledgments}

This work was supported by Sichuan University Scientific Research Foundation for Young Teachers, No. 2012SCU11066. This material is based in part on work reported (no. 0278-1896959) in 2011 IEEE Power Engineering and Automation Conference, which we would like to gratefully acknowledge. Prof. Mehdi Ahmadian, Prof. Valder Steffen and reviewers of this manuscript are also gratefully acknowledged for improving the quality of this article.

\section{References}

[1] P. Li, H.B. Dou and Z. Wang, Modeling for rotor-bearing system of hydroelectric machines and its nonlinear transient response, Journal of Tsinghua University (Sci and Tech) 38(6) (1998), 123-128 (in Chinese).

[2] R. Cardinali, Dynamic simulation of non-linear models of hydroelectric machinery, Mechanical Systems and Signal Processing 7(1) (1993), 29-44.

[3] T.W. Dimond, P.N. Sheth, P.E. Allaire and M. He, Identification methods and test results for tilting pad and fixed geometry journal bearing dynamic coefficients - A review, Shock and Vibration 16 (2009), 13-43.

[4] R. Nordmann and W. Diewald, Rotordynamics, in: Vibration and Oscillation of Hydraulic Machinery, H. Ohashi, ed., Brookfield, Avebury Technical, 1991, pp. 135-173.

[5] B.P. Bettig and R. Han, Modeling the lateral vibration of hydraulic turbine generator rotors, Journal of Vibration and Acoustics 121(3) (1999), 322-327.

[6] F.Z. Feng and F.L. Chu, Dynamic analysis of a hydraulic turbine unit, Mechanics of Structures and Machines 29(4) (2001), 505-531.

[7] L.F. Ma and X.Z. Zhang, Numerical simulation of nonlinear oil film forces of tilting-pad guide bearing in large hydro-unit, International Journal of Rotating Machinery 6(5) (2000), 345-353.

[8] X.H. Si, W.X. Lu and F.L. Chu, Lateral vibration of hydroelectric generating set with different supporting condition of thrust pad, Shock and Vibration 18 (2011), 317-331.

[9] H. Nelson, A finite rotating shaft element using Timoshenko beam theory, Journal of Mechanical Design 102(10) (1980), 793-803.

[10] J.R. Hutchinson, Shear coefficients for Timoshenko beam theory, Journal of Applied Mechanics 68(1) (2001), 87-92.

[11] B.C. Wen, J.L. Gu and S.B. Xia, Advanced Rotordynamics, China Machine Press, Beijing, 1999, (in Chinese).

[12] Y. Xu and Z.H. Li, Computational model for investigating the influence of unbalanced magnetic pull on the radial vibration of large hydro-turbine generators, Journal of Vibration and Acoustics-Transactions of the ASME 134(5) (2012), 051013.

[13] A. Muszynska, Rotordynamics, CRC Press, Boca Raton, FL, USA, 2005.

[14] J.W. Lund, Spring and damping coefficients for the tilting-pad journal bearing, ASLE Transactions 7(1964), 342-352.

[15] Z.H. Li, Y.Z. Ai and H.X. Shi, Optimal maintenance information system of Gezhouba hydro power plant, in: Power Engineering Society General Meeting, IEEE, Florida, 2007, pp. 1-5.

[16] N.R. Ma and Y.W. Tu, Operating experience of the Turbine guide bearings for Kaplan turbine, Huazhong Electric Power 2 (1990), 22-26 (in Chinese). 

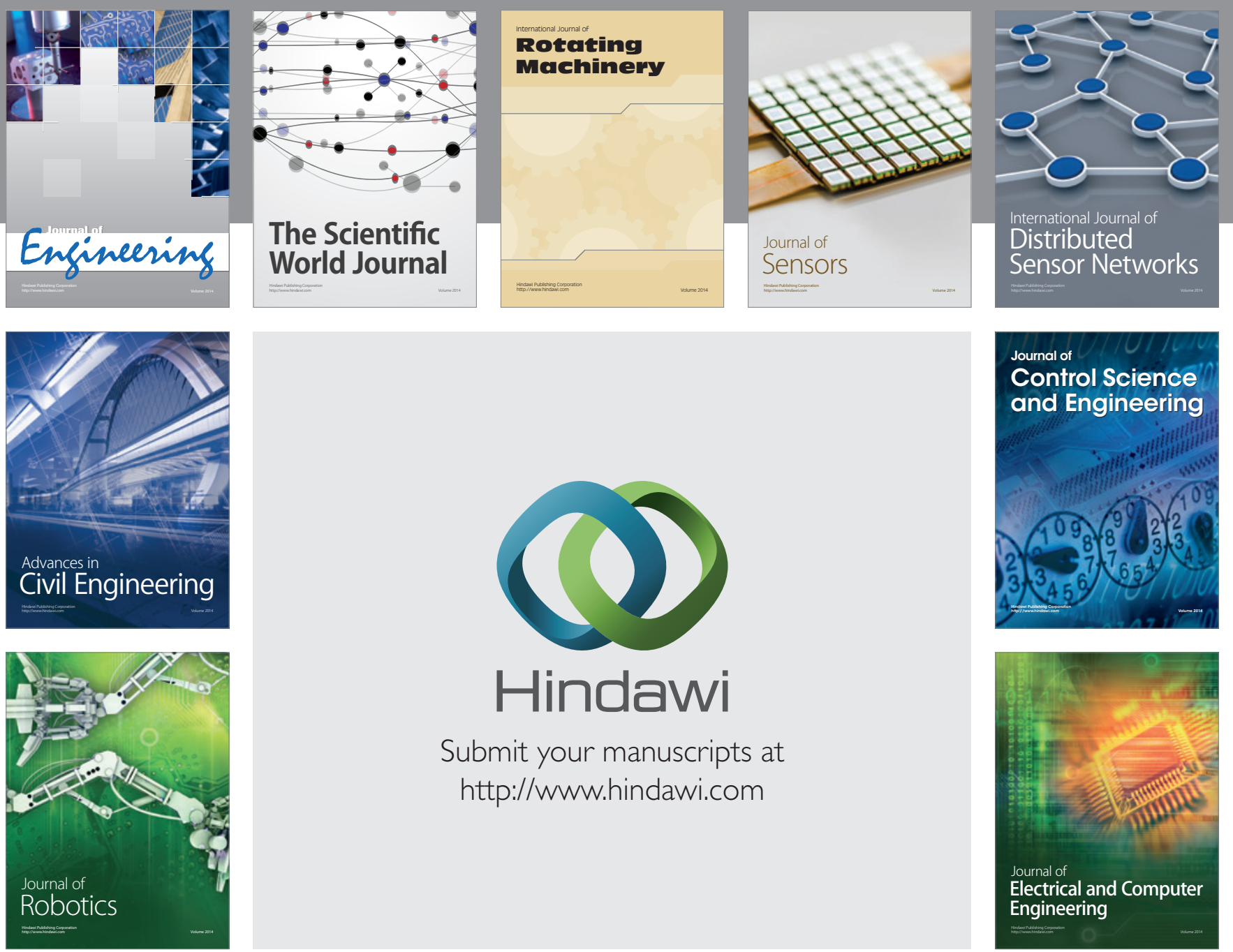

Submit your manuscripts at

http://www.hindawi.com
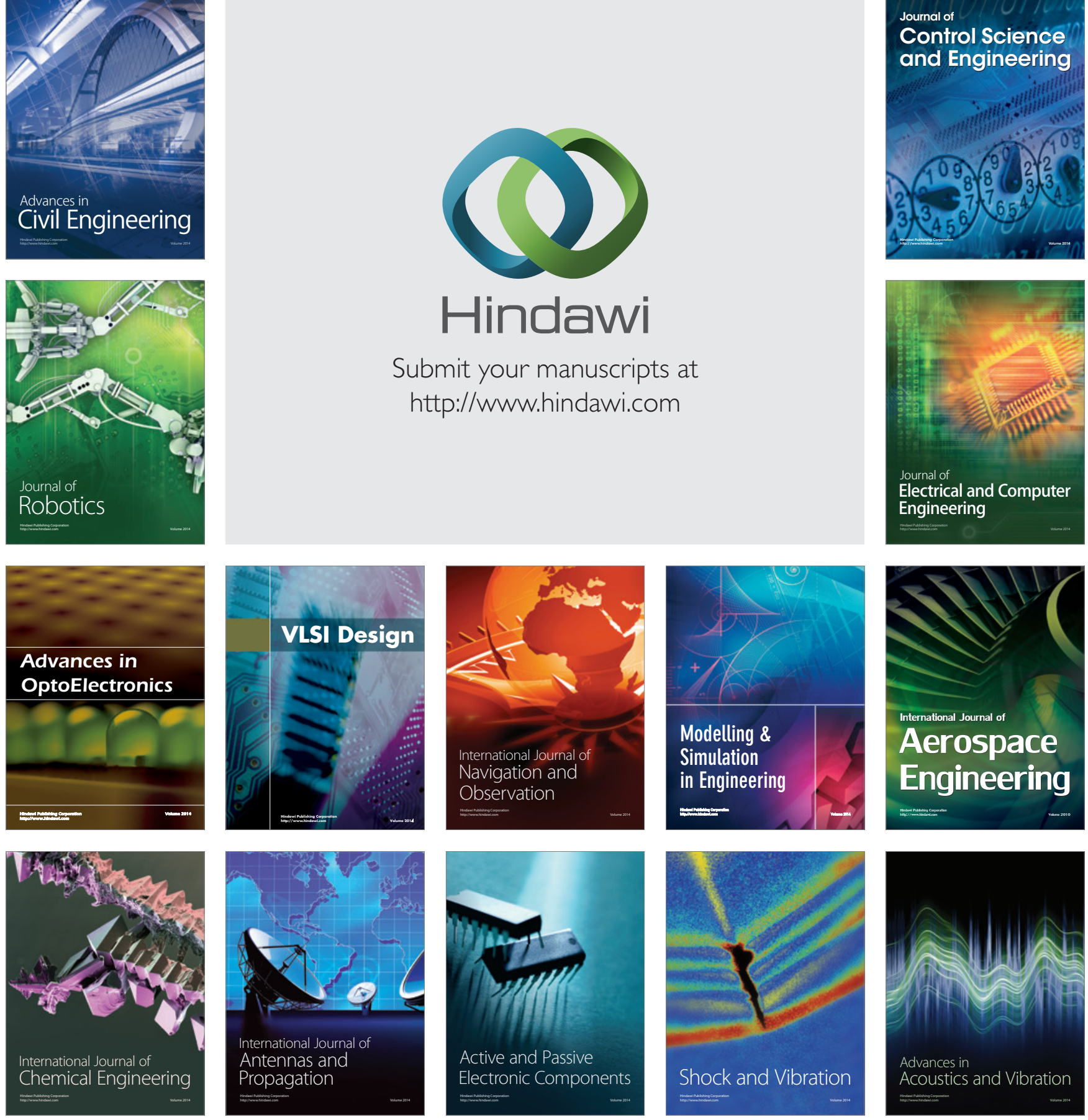